\title{
Needs and Wishes for the Future Exoskeleton: an Interview Study Among People With Spinal Cord Injury With Community-based Exoskeleton Experience
}

\section{Rosanne B. van Dijsseldonk ( $\sim$ r.vandijsseldonk@maartenskliniek.nl )}

Radboud University Medical Center https://orcid.org/0000-0002-4459-5435

Joke E. Vriezekolk

Sint Maartenskliniek

Noël L.W. Keijsers

Sint Maartenskliniek

Alexander C.H. Geurts

Radboudumc

Ilse J.W. van Nes

Sint Maartenskliniek

\section{Research}

Keywords: Spinal cord injury, Exoskeleton, User experience

Posted Date: December 28th, 2020

DOI: https://doi.org/10.21203/rs.3.rs-135258/v1

License: (c) (1) This work is licensed under a Creative Commons Attribution 4.0 International License. Read Full License 


\section{Abstract}

Background: Exoskeleton use by people with complete spinal cord injury ( $\mathrm{SCl}$ ) in daily life is still a challenge. To optimize its daily life use, a better understanding of the purpose of use and the accompanying improvements are needed. The perspective of experienced exoskeleton users provides an important contribution to the improvement of future exoskeletons.

Methods: Face-to-face semi-structured interviews were held with 13 people with SCl, who were experienced exoskeleton users. Interviews were audio-taped, transcribed and analysed thematically.

Results: Participants expressed three future purposes of exoskeleton use: for daily activities, exercise, and social interaction. Exoskeleton use during daily activities was the ultimate goal. Therefore, the future exoskeleton should be: easy to use, small and lightweight, tailor made, safe, comfortable, less distinctive, durable, and affordable. Improving the ease of use was relevant for all purposes, for all participants. The other suggestions for improvement varied depending on the purpose of use and the participant.

Conclusions: Increasingly more advanced improvements to future exoskeletons are needed to transition from an exercise purpose, to social interaction, and ultimately use during daily activities. In the current study, detailed suggestions for improvements from experienced exoskeleton users have been made. Only when multiple of these suggestions are adjusted, can the exoskeleton be used to its full potential.

\section{Background}

Spinal cord injury (SCl) is characterized by damage of the spinal cord that leads to (partial) loss of sensory, motor and autonomic functions below the lesion.[1] A complete SCl is characterized by paralysis of the muscles below the lesion level and, thus, recovery of walking capacity is unlikely, resulting in a lifetime reliance on a wheelchair.[2, 3] Further, people with $\mathrm{SCl}$ are also at risk for the occurrence of secondary health complications, such as bladder and bowel disorders, pressure ulcers, spasticity and pain, $[4,5]$ which are associated with a lower quality of life.[5] Recently, wearable exoskeletons (such as the ReWalk ${ }^{\mathrm{TM}}, \mathrm{Ekso}^{\mathrm{TM}}$, and Indego ${ }^{\circledR}$ exoskeleton) have appeared on the consumer market.[6] In the clinical setting, wearable exoskeletons are mainly used for training purposes and to enhance health benefits in people with complete SCl. The health benefits include less spasticity,[7, 8] improved bowel function,[9] and improved overall quality of life.[7] However, to preserve these health benefits, regular exoskeleton use is necessary. One approach to facilitate regular exoskeleton use is to ensure that wearable exoskeleton can be used at home and in the community.

Little is known about the use and experienced benefits of exoskeleton use in the community. To our knowledge, two studies investigated community exoskeleton use, namely in a community-based gym[10] or in and around home.[11] In both studies, the exoskeleton was mainly used for exercise purposes.[10, 11] Participants also emphasized the usefulness of an exoskeleton for social interaction.[10,11] The exoskeleton was rarely used to facilitate functional mobility during daily activities.[11] Study participants reported physical and psychosocial benefits after using the exoskeleton for approximately ten weeks[11] 
or one year[10]. They experienced less spasticity, less pain, better wound healing, improved bowel function, and improved mental wellbeing and increased energy to (socially) interact at eye-level.[10, 11] Although there seem to be physical and psychosocial benefits for exoskeleton users, it is unclear which barriers exoskeleton users experience in the home and community setting.

A few studies examined possible barriers and/or improvements of an exoskeleton in order to optimize its use. Reported areas of improvement were safety,[12-15] ease of use (including the need of a buddy,[12$14]$ need of upper extremity support,[13, 16] walking speed,[12, 14, 15] donning,[12-15] transportability, $[13,16]$ and energy demand[14]), costs,[14, 15], and comfort.[15] However, these suggested improvements are from researchers and individuals with no exoskeleton use experience.[12-16] The needs and wishes for the future exoskeleton from the perspective of people with $\mathrm{SCl}$ with community-based exoskeleton experience are still unclear. In addition, the various barriers people with $\mathrm{SCl}$ may experience when using the exoskeleton may vary depending on the purpose of its use (e.g., the need of a buddy might be a barrier for functional daily use, but not for social interaction at an eye-level).

To optimize the use of exoskeletons in daily life by people with complete $\mathrm{SCl}$, a better understanding of the purpose of use and accompanying improvements from the perspective of exoskeleton users are needed. The perspective of experienced users provides an important contribution to the improvement of future exoskeletons. These insights can inform future exoskeletons designs, and ultimately, can help to optimize community-based exoskeleton use. Therefore, the main research questions of this qualitative study were (1) for which purpose(s) would (potential) exoskeleton users like to use the exoskeleton in the future and (2) which improvements are needed to the current exoskeleton to facilitate these desired purpose(s) of use?

\section{Methods}

A qualitative study design involving in-depth semi-structured interviews with thematic analysis was chosen to address the research questions. All participants gave written informed consent in accordance with the Declaration of Helsinki. The medical ethics committee of Arnhem-Nijmegen determined that this study met the requirements for exemption from the Medical Ethics Committee review under the Dutch Medical Research Involving Human Subjects (2019-5637). The study was approved by the internal review board of the Sint Maartenskliniek.

\section{Participants and recruitment}

Adult people in the chronic phase (> 6 months) after a motor complete SCI (American Spinal Injury Association Impairment Scale (AIS) A or B) who used a wearable exoskeleton both in the clinical setting and community-setting, and had proficiency in the Dutch language were eligible. Eligible people who participated in our previous exoskeleton study (2016-2418 [11]) and who gave consent to be contacted for a follow-up study were approached. In addition, people in the Netherlands who purchased their own exoskeleton were contacted to participate. To our knowledge, this was the entire population of Dutch 
people with complete $\mathrm{SCl}$ who used an exoskeleton in the home and in the community setting. Potential participants were first approached by an e-mail containing the information letter. If they had no objections, they were contacted by telephone after one week of reflection and, if they wanted to participate, to schedule an interview appointment.

\section{Data collection}

Single face-to-face interviews were held at the participant's home or at the rehabilitation clinic, according to the preference of the participant. The interview guide consisted of semi-structured open-end questions (Fig. 1). Interviews were conducted by the first author (PhD candidate), with whom all participants were familiar from the previous study [11] and who received training to perform interviews. No other participants or researchers were present during the interview. As a sensitizer, each interview started with a short videoclip (+/- 1 minute) of the participant's final clinical training session with the exoskeleton before two-weeks of community use as part of the previous study.[11,17] Each interview was audio-recorded and transcribed verbatim for data analysis. During and after each interview, field notes were made. To ensure complete and transparent reporting, the Consolidated Criteria for Reporting Qualitative Research (COREQ) checklist was used.[18]

\section{Data analysis}

Data collection and data analysis was alternated and repetitively reflected on by two researchers (RvD and JV). Analysis of the data was done in an iterative process using thematic analysis. Transcripts were coded by the first author and discussed with the second author until consensus was reached. Initial open coding was performed by the first author, with labelling segments of the transcript close to the phrasing of the participant. Thereafter, codes were refined throughout the analysis and categorized into themes (axial coding). The core themes were based upon concepts extracted from literature.[11, 13, 15, 19] Memos were written and used during the analysis to help the researchers keep track of and reflect upon decisions made. To help interpret the findings, the field notes (from during and after the interview) were used. Data saturation was assumed if no new axial codes emerged from the analysis of the last two interviews. The final results (selective coding) were discussed with the first, second, and last author and adjusted if necessary, until consensus was reached. The software program ATLAS.ti was used to code the data and write the memos.

\section{Results}

Thirteen out of the sixteen contacted people participated in the study. Reasons for not participating were self-reported lack of sufficient community-based exoskeleton experience $(n=1)$, and limited time to participate $(n=2)$. A summary of the characteristics of the thirteen participants is given in Table 1. To ensure confidentiality, codes ( $\mathrm{P} 1^{\text {gender(age) }}, \mathrm{P} 2^{\text {gender(age) }}$ etc) were used instead of the participant's names when indicating from whom a quote originated. All participants were experienced exoskeleton users, who completed a clinical training period of approximately eight weeks (+/- 24 training sessions). They all used the ReWalk ${ }^{\text {TM }}$ exoskeleton in the home and community setting, the amount of community use ranged from 
three to more than 100 days). Three participants had purchased their own ReWalk ${ }^{\text {TM }}$ exoskeleton through crowdfunding and, thus, had much more community exoskeleton experience. In addition, six participants also had experience with another exoskeleton: the Indego $\AA$ exoskeleton in a clinical setting $(n=4,+/$ 3 years ago) and/or a research exoskeleton (i.e., March or Symbitron) in a lab setting ( $n=4,+/-1.5$ years ago). The time between the last community-based exoskeleton use and the interview ranged between one day and four years (median of two years). The duration of the interviews varied between 30 and 90 minutes.

Table 1

Participant characteristics.

\begin{tabular}{|ll|}
\hline & Total $(\mathbf{N}=\mathbf{1 3})$ \\
\hline Sex (male / female) & 7 / 6 \\
\hline Age (years), median [min - max] & 33 [25-52] \\
\hline Time post injury (years), median [min - max] & 8 [2-28] \\
\hline Neurological level of SCl (thoracic), median [min - max] & Th9 [Th4 - L1] \\
\hline Classification of SCl level (low (Th7-12) / high (Th1-6)) & 7 / 6 \\
\hline AIS* (A / B) & 12 / 1 \\
\hline$*$ AIS = American Spinal Injury Association Impairment Scale, Th = Thoracic, L = Lumbar \\
\hline
\end{tabular}

\section{Purpose of exoskeleton use}

Three future purposes of exoskeleton use were mentioned in the interviews, namely for daily activities, exercise, and social interaction. Table 2 provides an overview of the purposes of use with exemplary citations. The use during daily activities and for exercise was addressed in the interviews of all participants. Most participants wanted to use the future exoskeleton during daily life activities in which they now used the wheelchair, such as grocery shopping or driving a car. One person even expressed that he wanted to do all activities that he was able to before the $\mathrm{SCl}$, such as walking on the beach or running up the stairs. Participants also mentioned that they were interested in using the exoskeleton as an exercise device to stay fit and to stimulate health benefits. Furthermore, the use of the exoskeleton for social interaction (e.g., go to the pub or join in a group chat at a party with bar tables) was mentioned in almost all interviews. Some participants emphasized that for exercise and social interaction you do not need to purchase your own exoskeleton, but could share an exoskeleton with others as well. 
Table 2

Purposes of exoskeleton use with exemplary citations.

\section{Daily activities}

"As it is now, I would use the Rewalk for what I call physio sports. However, you would want to use it to do the things you used to be able to do, like getting up, driving a car, you'd put it on in the morning and keep it on all day. Possibly using it in combination with a wheelchair. It'd be nice though if you didn't need your wheelchair at all." P10 female(52)

"Ultimately, l'd like to be able to, for example, go for a walk on the beach with my daughters, or take them to an amusement park, and go on rides you actually have to go up steps for. Or at least that you can do things that you can't do right now because of that [wheel]chair." $\mathrm{P} 3^{\text {male(29) }}$

"Well, what I envision is a kind of exoskeleton that I can put on like a pair of trousers and that I can wear sitting in my wheelchair. So that, whenever I want to, I can get up or climb a flight of stairs, or whatever. [...] When I think of that, of what would be possible, a whole new world opens up." P11male(37)

\section{Exercise}

"The purpose of walking? Well, for me it's pushing back the boundaries. Each time, I try to walk further with it. That's great. First just a short distance, and then each time go a bit farther. Yeah, the goal is to push back the boundaries and see how far you can walk with a suit [= exoskeleton] like this." pgmale(49)

"But l'd like to use it in a rehabilitation centre, for example, to be able to walk. Preferably together with others, so you can learn from each other. For the health benefits, you know. I wouldn't use it so much for practical things, but more to improve my health, and not have to sit in my chair all day. That I can walk. That there's pressure on my joints. So that you can keep walking. That's why I think it's useful... to improve your health. I'd really like to use it for that." $\mathrm{P} 2^{\text {female(44) }}$

"Also for health reasons, of course. Having walked for about three and a half months I felt even fitter. Just bladder and bowel goes easier and, when you walk and move your hips a few times a week, it [your body] feels more comfortable, that sounds a bit weird, but yes that's what happens." P4 female(49)

Social interaction

"So that you can join in. For instance, I hate parties where they have standing tables. It'd be great to be able to just stand at a table like that. And with a bar stool nearby for support if necessary or to rest a bit, so that basically you don't stand out from the crowd. Yes, I think that's something that would make this kind of suit [= exoskeleton] very useful." $\mathrm{P} 7^{\mathrm{female}(30)}$

"I didn't want to think about that for a very long time. I told myself that it wasn't really important to me. But it's true that people really like to look at you at eye level. And they suddenly notice you again and say, hey, wow, I haven't seen you standing up like this before." P5 female(49)

\section{Motives for exoskeleton use}

In addition to the purposes of future exoskeleton use, three motives for exoskeleton use were clearly described in the interviews, namely societal importance, preserving autonomy, and fun. A few participants 
mentioned that for them, a motivation for exoskeleton use was the societal importance for further development.

P5 female(49): "I think it's something that's still in its infancy, that needs to be developed further. [...]. I think we should all do our bit to make that happen [...] it may actually be something that will be covered by health insurance in the future. Mainly for people who've recently suffered a spinal cord injury, I should think."

The use of the exoskeleton was frequently compared to wheelchair use, which is something this population uses on a daily basis. For example, some participants stressed that they are completely autonomous with their wheelchair, and that this level of independence is something they also wanted to pursue with the future exoskeleton, especially during daily activities.

P2 female(44): "Independence. I think that's the biggest one. I'm now too dependent on someone else or on devices, for safety, weight, all the things I can't do myself, over which l've no control now. Yes, independence. Not needing help with anything. That's it basically."

Contrary to wheelchair use, the motive for exoskeleton use was sometimes referred to as 'for fun', in which fun was an end in itself.

P7female(30): "Well.. it has to be fun. That it becomes less therapeutic. Sure, it will never be exactly the walking you were used to [before the injury], but I do think that it's very close, that you can just go for a nice stroll around the block."

\section{Improvements of exoskeleton}

To optimize the future exoskeleton, different improvements were suggested. Experienced exoskeleton users mentioned that their future exoskeleton is easy to use, small and lightweight, tailor made, safe, comfortable, less distinctive, durable, and affordable (figure 2). The most inner circle (i.e., easy to use) of figure 2 represents the desired improvement that was relevant for all purposes of use, for all participants. The circles further to the edge, represent improvements that are more person-dependent or dependent on the purpose of use. In the next sections, the different suggestions for improvement will be discussed.

\section{First circle: Easy to use}

Regardless for which purpose the future exoskeleton will be used, all participants mentioned that the future exoskeleton should be easier to use. Therefore, participants advised to improve the remote-control watch, balance, step and speed adjustments, and donning and doffing of the exoskeleton. Improvements to the remote-control watch were mentioned most frequently, for instance improving readability in sunlight and/or changing control options. Suggested control options were via speech or via touch (e.g., touching the thigh to walk and touching the bottom to sit down). Some participants suggested to relocate the control to the crutches, as long as the crutches are needed for balance support. This crutch-control would preferably include a small screen on which different speeds, step heights and step frequencies 
could be selected. In addition, participants mentioned that keeping balance with the exoskeleton (especially in stance) required much physical and mental effort. To be able to focus on other things, such as a conversation or use of kitchen utensils, balance improvements in the future exoskeleton were desired.

P11 male(37): "What's essential in that suit [= exoskeleton]? What can still be improved? Well, first of all, the balance. Not having to use crutches anymore is the most important thing. [...] That you don't constantly need to keep your balance; the suit should do that for you. That's what I think is crucial. Suppose I could walk the way I am doing now, but could keep my balance without needing crutches. That would immediately be a real functional improvement."

Step and speed adjustments were desired so that the exoskeleton could be used more broadly.

P9male(49): "It should have gears, like in a car. Or let's take a bike as an example. Let's say you have one, two, three positions and that the third position is the highest gear. This gear allows you to, for example, take a big step, or increase your pace. That works perfectly for outdoors. Position two would then be something just in between. And position one would reduce the length of your steps and their frequency. Then I think it could be used functionally in a home situation indoors."

In addition, some participants expressed the wish don and doff the exoskeleton, without needing several

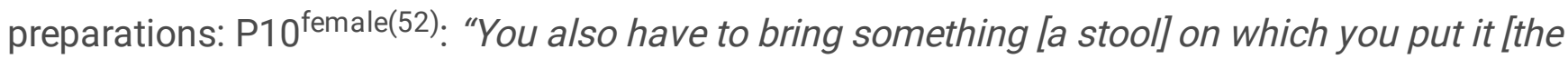

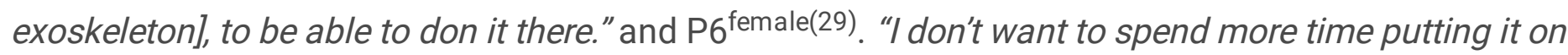
than that I'm actually using it."

\section{Second circle: Small, lightweight and tailor made}

The improvements in the second circle were mostly related to transporting the exoskeleton and adjusting the hardware to the body of the user. Participants agreed that they could not lift and transport the exoskeleton (in a convenient way) by themselves, due to the weight and the physical dimensions.

P8male(28): "But maybe it will make a difference when carrying it to and from the car. If I can move it myself, for example, in three sections instead of one big awkward lump for which I would need someone else to help me. [...] It wouldn't be so heavy then. Maybe it'll save some space. That would make me independent. I'd be very happy, even if it did take me five minutes longer to put it on."

In addition, participants mentioned that, especially due to the crutches, you need a lot of space. Therefore, some participants noted that their (wheelchair friendly) house was too cramped for exoskeleton use. A minority of the participants expressed the desire for an exoskeleton that is exactly tailored to their body, but can also be adjusted to different people, in case you want to share an exoskeleton.

P7female(30): "Normally you have enough space but now it feels mega small, because with that suit [= exoskeleton] and those crutches you're a bit like a big robot stomping around the house. It's not very 
pleasant. I hope that in the future they'll be able to develop suits that are as compact as possible, so that you can easily use [them] at home. It would be really great if I could do more things standing or walking."

\section{Third circle: Safe, comfortable and less distinctive}

Opinions on improving safety were diverse. Some participants considered the safety of the exoskeleton as very important and even something that - if not guaranteed - would lead to disuse, while others felt that using the exoskeleton was safe and the risks were similar to other activities.

P5 female(49) "Improvements that would make me use it regularly to move about? Well, most certainly an emergency stop. [...] Suppose getting up doesn't go well... if you could just press something in your crutches if things start to go wrong, and you'd be able to get back in the right position and slowly lower it, so that you go back to base [the sitting position], if I had had those options I think it would have been less scary for me."

$\mathrm{P} 4$ female(49) "In the end there is one thing you don't want and that is falling."

P9male(49): "Yes, you can fall, but you can also fall over in your wheelchair, you know. There's no danger of that. Zero, nil, nothing! It may happen that your crutch slips when you're walking somewhere and that it bounces a bit, but so what? If I go handcycling, another road users may not see me and I may get hit by a car, but that's a risk I'm willing to take."

Participants who desired an exoskeleton that could be worn all day, mentioned that it should be comfortable to wear and that, for example, a battery on your back would be inconvenient in order to sit comfortably (anywhere).

P11 male(37): "It's just a hassle with that thing. You're completely constricted, even when sitting. You can't really move well in it. So I could sit in the pub in it, but I wouldn't feel very relaxed."

Although most participants focused more on the functionality of the exoskeleton, some participants expressed the wish to make the exoskeleton's appearance as close to normal as possible, so that they don't attract (extra) attention in public situations (e.g., less visible and less noisy).

$\mathrm{P} 1$ male(27): "That it [the walking pattern] looks natural. So that people don't think you're some kind of robot. I think you could easily do this with the latest software."

\section{Fourth circle: Affordable and durable}

Most participants indicated that the current exoskeletons are too expensive, especially with respect to what it is currently capable of. Therefore, almost all participants focused on improvements to its functionality. However, a minority mentioned that not all improvements were necessary and one participant pointed out that the exoskeleton should have fewer functionalities, so that it could be marketed cheaper and become accessible to a larger group. 
P1 male(27): "I think you first need to focus on affordability and, of course, functionality, particularly if you want to stimulate further development. After all, it's a very specific target group."

In addition, only a few participants mentioned improvements about the durability of the exoskeleton.

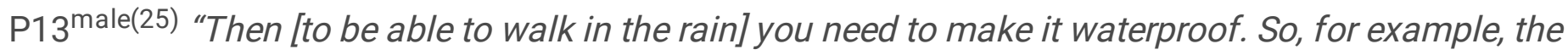
bearings need to be well sealed, and you need to use corrosion-resistant material. Like mobile phones. All mobile phones are water resistant nowadays... So that means a backpack... but that will all drive up the price."

\section{Discussion}

In this qualitative study, experienced exoskeleton users expressed three future purposes of exoskeleton use: for exercise, social interaction, and daily activities. Using the current exoskeleton for exercise is already possible[11, 20,21] and only a few improvements to make it easier to use were desired for this purpose. To facilitate social interaction, besides easier to use, the exoskeleton should also be small and lightweight, tailored to the person, and for some people less distinctive. For the ultimate purpose of future exoskeleton use, namely during daily activities, the most improvements were needed.

The extent of improvements needed for daily activities varied between participants, possibly reflecting participants who kept their answers closer to nowadays reality (i.e., feasible with the current technological knowledge) and participants who were willing/dared to describe their ultimate future exoskeleton ('the sky is the limit'). For instance, improvements to the comfort level varied from improving seating comfort in a (wheel)chair, to a level where the exoskeleton never has to be taken off again (i.e., a lifetime of 24/7 use, including wearing it in bed and while showering). Although participants who described the 'sky-is-thelimit' exoskeleton may have given unrealistic answers from a designer's perspective, there is great value in these answers because they provide insight into what is ultimately pursued. Exoskeleton use during daily activities was often discussed in the context of participants current, wheelchair-bound, execution of daily activities. Contrary to wheelchair use, the motive for exoskeleton use was also referred to as 'for fun'. Something that will rarely be stated about the wheelchair, which was only considered an assistive device.

Although we (partly) found similar areas of improvement as previous studies, the relevance and content of these areas of improvement sometimes differed. A previous survey study concluded that safety, affordability, ease of use, and comfort were considered the most important areas of improvement[15]. The relevance of improving the ease of use was supported by our findings (i.e., inner circle in Fig. 2). However, the other areas of improvement were more purpose and person dependent, suggesting that they were considered less relevant. Especially for safety, the relatively low relevance of improvement was not expected from both a clinical and a design perspective. In addition, the content within an area of improvement sometimes differed with previous findings. For example, the current study revealed specific improvements to the remote control watch (e.g., readability), to make the exoskeleton easier to use, 
instead of the earlier reported improvements to the comprehensive concept of 'ease of use' of the exoskeleton.[15] In addition, being able to adjust the speed (i.e., accelerate and decelerate) while using the exoskeleton in various situations was desired, instead of maximizing the speed of the exoskeleton itself. $[12,14,15]$ Also a reduction in the number of preparations needed before exoskeleton use (e.g., placing the exoskeleton on a stool) was desired, whereas, once these preparations were made, donning and doffing the exoskeleton was not an issue. These detailed, yet very important, improvements have hardly been discussed in previous studies.

The discrepancy compared to previous studies could be attributed to the distinct enrolled (patient) populations. The suggested improvements in previous studies are from researchers and individuals who have not used the exoskeleton.[12-16] Only people with exoskeleton experience (outside the clinical setting) will be able to mention detailed, yet very important, improvements for home and community use (such as to the remote control watch). In addition, all participants involved in this study can be seen as 'early adopters', meaning that they were already willing to use these not (yet) fully developed exoskeletons. However, it is important to keep in mind that the (needs and) requirements of these early adopters, may differ from the ultimate target group of the exoskeleton (i.e., the general SCI population). Nevertheless, also in these early adopters, multiple improvements were mentioned before the technology will become mainstream for a larger population.

\section{Strengths and limitations}

A strength of the current study is that almost the entire population of Dutch people with a complete $\mathrm{SCl}$ who used an exoskeleton in the home and in the community setting were interviewed and that data saturation was met. However, the community exoskeleton use was relatively long ago for some participants, which could have led to recall bias. To recall memories, and, thus, minimize the recall bias, a video of the participants own ReWalk ${ }^{\text {Tm }}$ exoskeleton use was shown prior to the interview. Although participants in the current study were particularly experienced with the ReWalk ${ }^{\mathrm{TM}}$ exoskeleton, we expect that the purposes of use, areas of improvement and, to a large extent, the relevance of the improvements are generalisable to other wearable exoskeletons that rely on upper extremity support. Caution apply to the remote control watch and the 'small and lightweight'-suggestion is advised, because these aspects differ between the most commonly used exoskeletons (i.e. ReWalk ${ }^{\mathrm{TM}}$, Indego ${ }^{\circledR}$, and Ekso bionics ${ }^{\mathrm{TM}}$ ).[2224]

\section{Conclusions}

Experienced exoskeleton users expressed three future purposes of exoskeleton use: for daily activities, exercise, and social interaction. Increasingly more advanced improvements of future exoskeletons are needed to transition from an exercise purpose, to social interaction, and ultimately use during daily activities. In the current study, detailed suggestions for improvements from experienced exoskeleton users have been made. Only when multiple of these suggestions are adjusted, the exoskeleton can be used to its full potential. 


\section{Abbreviations}

AIS: American spinal injury association impairment scale; COREQ: Consolidated Criteria for Reporting Qualitative Research; L: Lumbar; SCl: Spinal cord injury; Th: Thoracic

\section{Declarations}

\section{Acknowledgements:}

The authors would like to thank the persons with spinal cord injury for their participation in this study. We acknowledge H. Rijken, J. van Eupen, C. Tatu, and P. Koomen for providing the exoskeleton training sessions to all participants.

\section{Funding:}

This work is part of the research program Wearable Robotics with project number P16-05, which is (partly) financed by the Dutch Research Council (NWO).

\section{Availability of data and material:}

All data associated with this study are present in the paper.

\section{Ethics approval and consent to participate:}

All participants gave written informed consent in accordance with the Declaration of Helsinki. The study was approved by the medical ethics committee of Arnhem-Nijmegen (2019-5637) and the internal review board of the Sint Maartenskliniek.

\section{Competing interests:}

The authors declare that the research was conducted in the absence of any commercial or financial relationships that could be construed as a potential conflict of interest.

\section{Consent for publication:}

Not applicable.

\section{Authors' contributions:}

$\mathrm{RvD}, \mathrm{JV}$ and NK were involved in the design of the study and development of the interview guide. IvN was responsible for patient recruitment. RvD performed the interviews. RvD and JV contributed to the analysis, interpretation of the data, drafting the manuscript, and designing the figures. NK, IvN, and AG supervised the project. All authors edited and approved the final manuscript.

\section{References}


1. Nas K, Yazmalar L, Şah V, Aydin A, Öneş K. Rehabilitation of spinal cord injuries. World J Orthop. 2015;6:8-16.

2. Burns AS, Marino RJ, Flanders AE, Flett $H$. Clinical diagnosis and prognosis following spinal cord injury. 1st edition. Elsevier B.V.; 2012. doi:10.1016/B978-0-444-52137-8.00003-6.

3. Kay ED, Deutsch A, Wuermser LA. Predicting Walking at Discharge From Inpatient Rehabilitation After a Traumatic Spinal Cord Injury. Arch Phys Med Rehabil. 2007;88:745-50.

doi:10.1016/j.apmr.2007.03.013

4. Jensen MP, Truitt AR, Schomer KG, Yorkston KM, Baylor C, Molton IR. Frequency and age effects of secondary health conditions in individuals with spinal cord injury: a scoping review. Spinal Cord. 2013;51:882-92. doi:10.1038/sc.2013.112.

5. Adriaansen J, Ruijs L, Koppenhagen C, Asbeck F, Snoek G, Kuppevelt D, et al. Secondary health conditions and quality of life in persons living with spinal cord injury for at least ten years. J Rehabil Med. 2016;48:853-60. doi:10.2340/16501977-2166.

6. Shi D, Zhang W, Zhang W, Ding X. A Review on Lower Limb Rehabilitation Exoskeleton Robots. Chinese J Mech Eng (English Ed. 2019;32. doi:10.1186/s10033-019-0389-8.

7. Baunsgaard CB, Nissen UV, Brust AK, Frotzler A, Ribeill C, Kalke YB, et al. Exoskeleton gait training after spinal cord injury: An exploratory study on secondary health conditions. J Rehabil Med. 2018;50:806-13. doi: 10.2340/16501977-2372

8. Miller LE, Zimmermann AK, Herbert WG. Clinical effectiveness and safety of powered exoskeletonassisted walking in patients with spinal cord injury: systematic review with meta-analysis. Med Devices Evidenve Res. 2016;22:455-66. doi: 10.2147/MDER.S103102

9. Chun A, Asselin PK, Knezevic S, Kornfeld S, Bauman WA, Korsten MA, et al. Changes in bowel function following exoskeletal-assisted walking in persons with spinal cord injury: an observational pilot study. Spinal Cord. 2020;58:459-66. doi:10.1038/s41393-019-0392-z.

10. Cahill A, Ginley OM, Bertrand C, Lennon O. Gym-based exoskeleton walking: A preliminary exploration of non-ambulatory end-user perspectives. Disabil Health J. 2018;11:478-85. doi:10.1016/j.dhjo.2018.01.004.

11. van Dijsseldonk RB, van Nes IJW, Geurts ACH, Keijsers NLW. Exoskeleton home and community use in people with complete spinal cord injury. Sci Rep. 2020;:1-8. doi:10.1038/s41598-020-72397-6.

12. Gorgey AS. Robotic exoskeletons: The current pros and cons. World J Orthop. 2018;9:112-9. doi: 10.5312/wjo.v9.i9.112

13. Kandilakis C, Sasso-Lance E. Exoskeletons for Personal Use After Spinal Cord Injury. Arch Phys Med Rehabil. 2019. doi:10.1016/j.apmr.2019.05.028.

14. Palermo AE, Maher JL, Baunsgaard CB, Nash MS. Clinician-Focused Overview of Bionic Exoskeleton Use After Spinal Cord Injury. Top Spinal Cord Inj Rehabil. 2017;23:234-44. doi: 10.1310/sci2303-234

15. Wolff J, Parker C, Borisoff J, Ben Mortensen W, Mattie J. A survey of stakeholder perspectives on a proposed combined exoskeleton-wheelchair technology. J Neuroeng Rehabil. 2014;11:1-10. doi: 10.1186/1743-0003-11-169

Page $13 / 16$ 
16. Fritz H, Patzer D, Galen SS. Robotic exoskeletons for reengaging in everyday activities: promises, pitfalls, and opportunities. Disabil Rehabil. 2017;41:560-3. doi:10.1080/09638288.2017.1398786.

17. van Dijsseldonk RB, Rijken $H$, van Nes IJW, van de Meent $H$, Keijsers NLW. A Framework for Measuring the Progress in Exoskeleton Skills in People with Complete Spinal Cord Injury. Front Neurosci. 2017;11 December:1-12. doi:10.3389/fnins.2017.00699.

18. Tong A, Sainsbury P, Craig J. Consolidated criteria for reporting qualitative research (COREQ): A 32item checklist for interviews and focus groups. Int J Qual Heal Care. 2007;19:349-57. doi: 10.1093/intqhc/mzm042

19. Demers L, Weiss-Lambrou R, Ska B. The Quebec User Evaluation of Satisfaction with Assistive Technology (QUEST 2.0): An overview and recent progress. Technol Disabil. 2002;14:101-5.

20. Kolakowsky-Hayner SA. Safety and Feasibility of using the EksoTM Bionic Exoskeleton to Aid Ambulation after Spinal Cord Injury. J Spine. 2013;4. doi:10.4172/2165-7939.S4-003.

21. Gagnon DH, Escalona MJ, Vermette M, Carvalho LP, Karelis AD, Duclos C, et al. Locomotor training using an overground robotic exoskeleton in long-term manual wheelchair users with a chronic spinal cord injury living in the community: Lessons learned from a feasibility study in terms of recruitment, attendance, learnability, performa. J Neuroeng Rehabil. 2018;15:1-12. doi: 10.1186/s12984-0180354-2

22. Food and Drug Administration. Indego® - K173530. 2018;:1-12. https://www.accessdata.fda.gov/cdrh_docs/pdf17/K173530.pdf. Accessed 10 Oct 2019.

23. Food and Drug Administration. ReWalk ${ }^{\mathrm{TM}}$ P6.0 - K200032. 2020;:1-6. doi:10.1017/CBO9781107415324.004.

24. Food and Drug Administration. Ekso ${ }^{\mathrm{TM}}$ and Ekso GT ${ }^{\mathrm{TM}}$ - K143690. 2016. doi:10.4324/9780203128176-6.

\section{Figures}


Question 1: $\quad$ Could you describe the first day you used the exoskeleton at home or outside the clinic?

Question 2: Could you describe the last time you used the exoskeleton at home or outside the clinic?

Question 3: $\quad$ Suppose you would use the exoskeleton every day, for example like putting in your contact lenses every morning or wearing your glasses, what should the exoskeleton look like to you?

Question 4: For which activities would you like to use that exoskeleton? What would the purpose of the exoskeleton use be?

Question 5: Which changes to the current exoskeleton are needed for that purpose?

Question 6: Looking back at the exoskeleton you used, what do you think are the most important changes that are needed?

\section{Figure 1}

A short version of the interview guide.

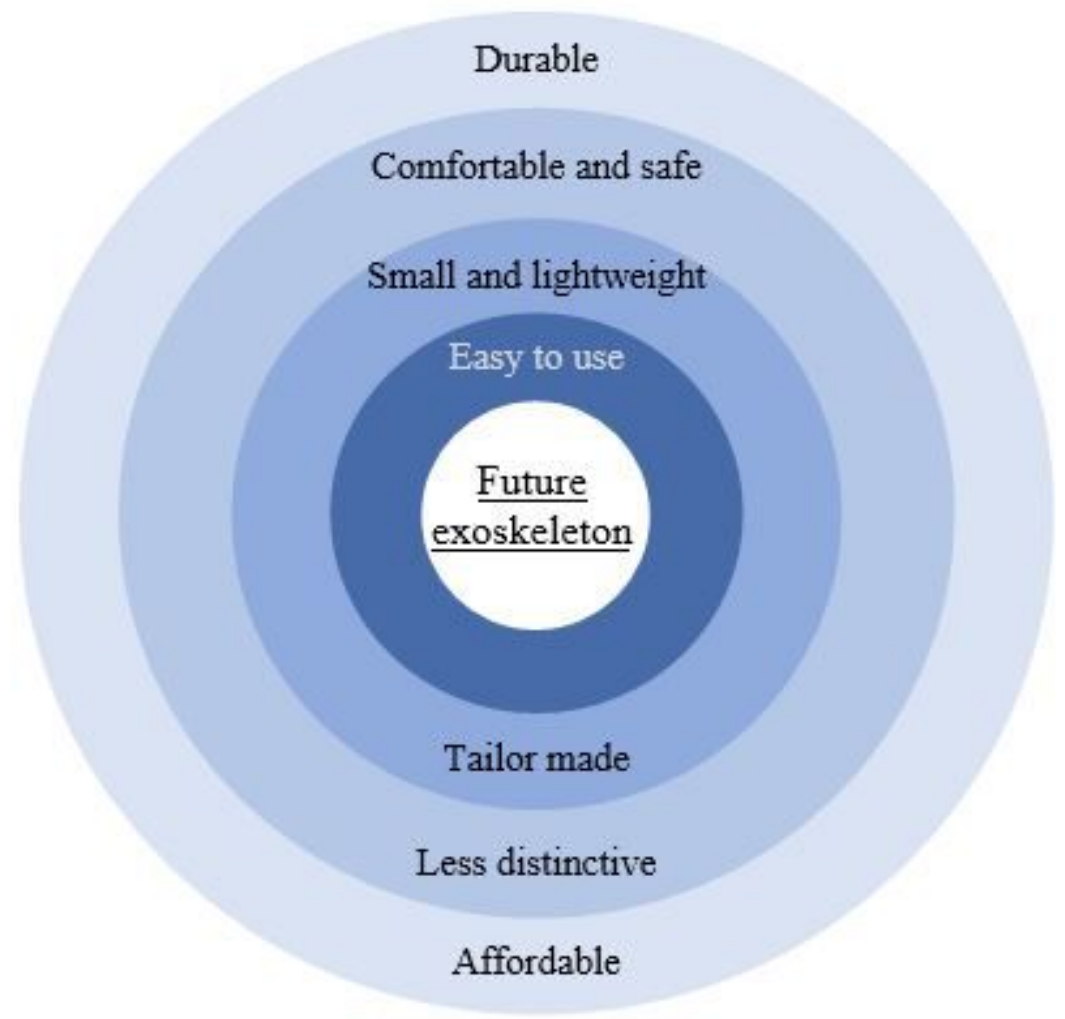

Figure 2 
Exoskeleton improvements. Darker shades circles are relevant for all purposes of use and for all participants, whereas lighter shades circles are more person-dependent and dependent of the purpose of use. 